\title{
Prospective evaluation of neutrophil autoantibodies in 500 consecutive patients with inflammatory bowel disease
}

\author{
Hugh Freeman mD, Brenda Roeck RT, Dana Devine PhD, Cedric CARTer mD
}

\begin{abstract}
H Freeman, B Roeck, D Devine, C Carter. Prospective evaluation of neutrophil autoantibodies in 500 consecutive patients with inflammatory bowel disease. Can J Gastroenterol 1997;11(3):203-207. Previous studies have shown antineutrophil cytoplasmic autoantibodies (ANCA) in patients with inflammatory bowel disease (IBD). A particular subclass, the so-called 'atypical' (perinuclear) p-ANCA type, occurs in the majority of patients with ulcerative colitis. The purpose of this prospective study was to assess, in a blinded fashion, this 'subclinical' serological marker in a consecutive series of IBD patients. Five hundred patients were evaluated, including 247 patients with ulcerative colitis and 253 with Crohn's disease involving the small and/or large intestine. Overall, 194 (38.8\%) of all patients with IBD were positive, including $164(66.3 \%)$ with ulcerative colitis and 30 (11.9\%) with Crohn's disease. Except for coexistent sclerosing cholangitis, no other clinical or laboratory variable had an effect on the rate of ANCA detection. This is the largest single study of ANCA in patients with IBD and the only study to provide data solely from a single Canadian centre. Results emphasize the immunopathological differences between ulcerative colitis and Crohn's disease, and indicate that both disorders are heterogeneous inflammatory disease processes.
\end{abstract}

Key Words: Antineutrophil cytoplasmic antibody, Atypical perinuclear antineutrophil cytoplasmic antibody, Inflammatory bowel disease, Ischemic/infectious colitis, Serum antibody, Serum marker

\section{Évaluation prospective d'auto-anticorps neutrophiles dans une série de 500 patients présentant une maladie inflammatoire de l'intestin}

\begin{abstract}
RÉSUMÉ : Des études antérieures ont mis en évidence la présence d'auto-anticorps cytoplasmiques anti-neutrophiles (ANCA) chez les patients souffrant d'une maladie inflammatoire de l'intestin (MII). Une sous-classe particulière, le type p-ANCA soi-disant « atypique » (périnucléaire), existe chez la plupart des patients souffrant de colite ulcéreuse. L'objectif de cette étude prospective était d'examiner, en aveugle, ce marqueur sérologique « sous-clinique » dans une série de patients souffrant d'une MII. On a évalué 500 patients, dont 247 atteints de colite ulcéreuse et 253 atteints d'une maladie de Crohn touchant le gros intestin et l'intestin grêle. Globalement, 194 (38,8 \%) de tous les patients atteints d'une MII étaient positifs, y compris 164 (66,3\%) des patients atteints d'une colite ulcéreuse et 30 (11,9\%) atteints d'une maladie de Crohn. À l'exception d'une cholangite sclérosante concomitante, aucune autre variable clinique ou de laboratoire n'a eu d'effet sur le taux de détection des ANCA. Cette étude est la plus vaste étude menée sur les ANCA chez les patients atteints de MII, et la seule étude à fournir des données provenant d'un seul établissement canadien. Ces résultats confirment les différences immunopathologiques existant entre la colite ulcéreuse et la maladie de Crohn, et montrent que ces deux affections sont des processus morbides inflammatoires hétérogènes.
\end{abstract}

A ntineutrophil cytoplasmic autoantibodies (ANCA) have been useful as a diagnostic aid in patients with necrotizing vasculitis and glomerulonephritis $(1,2)$. Recently a specific subclass of ANCA was also recognized in patients with inflammatory bowel disorders, particularly in those with idiopathic ulcerative colitis (3-5). While assays for ANCA are simple, inexpensive and reproducible (5), their utility in large series of patients with inflammatory bowel disease (IBD) still requires definition.

Subclasses of ANCA were initially based on the pattern 
of indirect immunofluorescence using alcohol-fixed neutrophils. These included a diffuse cytoplasmic pattern (c-ANCA) and a perinuclear pattern ( $\mathrm{p}$-ANCA). $\mathrm{p}$-ANCA is thought to be due to redistribution of antigen (myeloperoxidase and, possibly, other neutrophil granule components) towards the nucleus during alcohol fixation. A third subclass of ANCA, described as an 'atypical' form of $\mathrm{p}$-ANCA not reactive with myeloperoxidase $(5,6)$, occurs mainly in patients with idiopathic ulcerative colitis and primary sclerosing cholangitis $(7,8)$; different studies have documented a wide range of positive results - from about $25 \%$ to more than $80 \%(6-21)$.

Although detection of atypical p-ANCA may have diagnostic value in IBD patients, $\mathrm{p}$-ANCA has only been examined in limited numbers of patients in each reported series. In some studies, serum samples freeze-stored for variable periods were used, possibly altering results. Some of the earlier investigations provided data on ANCA serology from several countries. Except for some initial studies with a limited number of patients done in Los Angeles and including patient sera from Calgary $(6,12)$, this report is not only the largest in the literature, but provides data solely from a Canadian population centre. In the present evaluation, 'blinded' fresh serum samples from 500 consecutive patients with ulcerative colitis or Crohn's disease were examined for ANCA and, specifically, atypical p-ANCA. The results emphasize the immunopathological differences between ulcerative colitis and Crohn's disease.

\section{PATIENTS AND METHODS}

Patient groups: Patients had an established diagnosis of idiopathic ulcerative colitis or Crohn's disease in the small and/or large intestine based on clinical, radiologic, endoscopic and histological criteria, as well as based on negative microbiological studies (22).

There were 247 ulcerative colitis patients. Diagnosis was based on colonoscopic and histological studies demonstrating a diffuse mucosal inflammatory process in the rectum extending proximally within the colon. Radiologic studies were also often done to exclude small intestinal, particularly ileal, disease. Regarding disease extent and site, colitis was classified as, first, 'distal disease' if inflammatory changes extended from the anus but were localized in the distal $60 \mathrm{~cm}$ alone based on colonoscopic evaluation and mucosal biopsy; or, second, 'extensive disease' if inflammatory changes were over $60 \mathrm{~cm}$. In all patients with distal disease that was confined to the distal $60 \mathrm{~cm}$, biopsies were done to document the presence of normal proximal colonic mucosa.

There were 253 Crohn's disease patients. Diagnosis was usually based on colonoscopic and/or histological studies showing a segmental or patchy inflammatory process within the colorectum, distal small intestine or both, often with histological features of a focal and/or granulomatous inflammatory process. In addition, endoscopic mucosal biopsy, radiologic evaluation or both of the upper gastrointestinal tract were usually done. Radiologic and endoscopic data, as well as histological changes of inflammatory disease, were used.
When available, surgical materials were also reviewed from patients with either ulcerative colitis or Crohn's disease. All patient serological samples for this prospective study were collected in a consecutive fashion with no exclusions or refusals from October 1994 to December 1995; over $95 \%$ of samples were from out-patients rather than hospitalized patients. The clinician investigator was blinded to the results of serological studies for ANCA.

Control groups: Thirty-two additional patients, between 20 and 50 years old, served as controls for this study. Controls included 16 with abdominal pain and diarrhea; in these, endoscopic, radiologic and histological evaluation of the upper and lower gastrointestinal tracts were normal and microbiological studies were negative. Another 15 controls had infectious colitis; in all, typical endoscopic and histological features of a self-limited form of colitis were present, with a positive microbiological study for an established enteric pathogenic bacteria (Campylobacter and Yersinia species). A single patient with a focal segment of ischemic large intestine associated with cocaine use was included. The segment was resected.

Laboratory studies: For each patient, all blood samples were collected into vacutainer glass tubes (Becton Dickinson, New Jersey) by a laboratory technologist without knowledge of the patient's clinical history, investigation results or diagnosis. Blood was also obtained for hematological studies (hemoglobin, white blood cell count and platelet count), an erythrocyte sedimentation rate, antinuclear antibodies, liver chemistry tests (aspartate aminotransferase, alanine aminotransferase and alkaline phosphatase), serum iron studies and serum proteins, including serum albumin.

Finally, blood samples were collected into vacutainer glass tubes, allowed to clot at room temperature and used for detection of ANCA via two methods: ANCA immunofluorescence and ELISA. Serological studies for ANCA were done by a single laboratory technologist blinded to the clinical details and diagnosis. If ANCA immunofluorescence was positive, ANCA ELISA was done for autoantibodies to myeloperoxidase and serine protease 3 (PR3). As reported elsewhere with coded sera examined in a blinded fashion (3), excellent agreement was present between immunofluorescence and ELISA results.

ANCA immunofluorescence: Indirect immunofluorescence for c-ANCA, p-ANCA and atypical p-ANCA were detected with a standardized fluorescent antibody detection method $(6,7,23)$, using a proprietary kit purchased from a commercial supplier (Inova Diagnostics Inc, California). Slides were supplied with an adherent layer of cultured human neutrophils. The culture conditions were designed to ensure stability and strong expression of the primary cytoplasmic granules. The adherent neutrophils were fixed by the manufacturer with either ethanol or formalin. The primary screen for c-ANCA or p-ANCA involved incubation of serum at a 1:20 dilution in phosphate-buffered saline with ethanol-fixed slides for $25 \mathrm{mins}$ at room temperature followed by a 5 min wash with phosphate-buffered saline. This was followed by another $25 \mathrm{~min}$ incubation using affinity purified 
antihuman immunoglobulin (Ig) $\mathrm{G}$ with a fluorescent tag and a further $5 \mathrm{~min}$ wash with phosphate-buffered saline. Coverslips were then applied to the slides and these were examined with a fluorescent microscope at 500x magnification.

Positive ANCA cellular fluorescence was recorded and the distribution pattern of fluorescence designated as c-ANCA, p-ANCA or atypical p-ANCA (as shown in Figure 1). c-ANCA-positive sera were also titred. Sera positive for either $\mathrm{p}$-ANCA or atypical p-ANCA were further evaluated using formalin-fixed slides because this destroys nuclear antigens and atypical p-ANCA. In addition, formalin fixes both c-ANCA and p-ANCA antigens in the cytoplasm so that a false positive pattern will not be observed. Antinuclear antibody-positive sera were excluded because these may mimic ANCA.

ELISA assays: ANCA ELISA assays were performed with a standardized method $(6,7)$ using commercial kits (QuantaLite MPO and PR3 ELISA, Inova Diagnostics Inc). The test kits utilize microtitration strips containing wells coated with either proteinase-3, a primary antigen related to the $c$ ANCA pattern, or myeloperoxidase, a primary antigen associated with the p-ANCA pattern. Diluted serum was applied and incubated. If specific antibodies are present, binding to the wells occurs. Unbound material is initially removed by washing, and bound antibody detected by adding enzymelabelled antihuman $\operatorname{IgG}$, followed by a second washing, then incubated with a nitrophenol substrate. Wells with proteinase-3 or myeloperoxidase antibodies are quantitated by a colourimetric method.

\section{RESULTS}

Control subjects: All 16 patients in this evaluation with no detectable gastrointestinal disease, and all 15 patients with confirmed infectious colitis as well as a single patient with cocaine-associated ischemic colitis, had no neutrophil autoantibodies detected based on either immunofluorescent or ELISA assay detection methods.

IBD patients: As shown in Table 1, 194 of 500 patients with IBD (38.8\%) had atypical p-ANCA detected in serum samples with ELISA confirmation. A total of 164 of 247 patients with ulcerative colitis $(66.4 \%)$ were positive for atypical $p$ ANCA, and 30 of 253 patients with Crohn's disease (11.9\%) were positive for atypical $\mathrm{p}$-ANCA.

Specific historical and clinical variables in patients with either ulcerative colitis and Crohn's disease had no apparent effect on the rate of detection of atypical p-ANCA, including age at the time of initial diagnosis, sex, prior surgery, prior medication use or medical treatment, type of medication used, duration of disease and familial history of IBD (either ulcerative colitis or Crohn's disease). However, in this study, all patients with concomitant primary sclerosing cholangitis, radiologically defined, had a positive atypical p-ANCA $(7,8,10,14,15,21,23)$. Finally, no correlation with other laboratory test results were detected including hemogram (level of hemoglobin, white blood cell count and platelet count), erythrocyte sedimentation rate, serum iron or protein levels, including serum albumin.

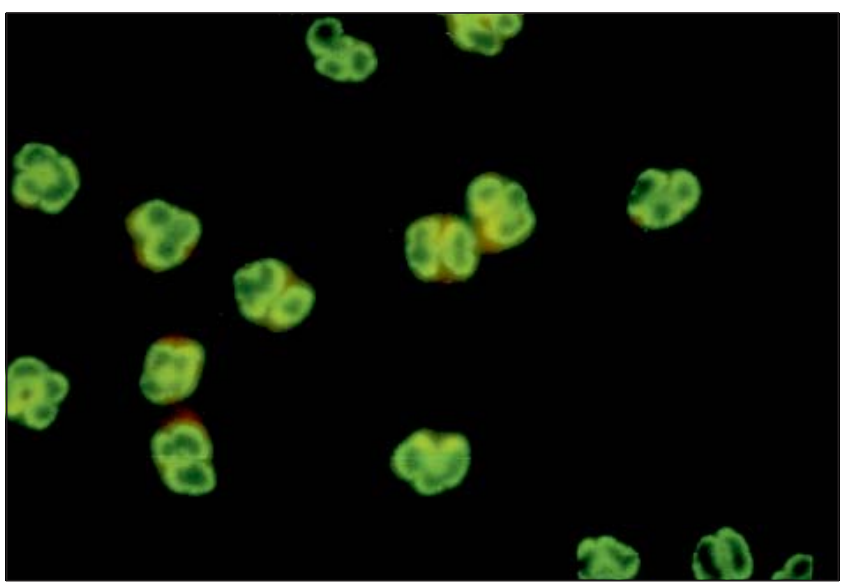

Figure 1) Representative immunofluorescent photomicrograph showing 'atypical' perinuclear antineutrophil cytoplasmic autoantibodies from a patient with ulcerative colitis. The patient serum in this alcohol-fixed slide appears completely negative on a formalin-fixed slide. Reproduced with permission from Inova Diagnostics Inc, San Diego, California

\section{TABLE 1}

Antineutrophil cytoplasmic autoantibody (ANCA) serology in inflammatory bowel disease

\begin{tabular}{lcc}
\hline Disease/site & ANCA-negative & ANCA-positive (\%) \\
\cline { 1 - 1 } Ulcerative colitis & & \\
$\quad$ Distal disease & 48 & $83(63.4)$ \\
Extensive disease & 35 & $164(69.8)$ \\
$\quad$ Total & 83 & \\
Crohn's disease & & $4(5.8)$ \\
$\quad$ Small bowel alone & 65 & $10(18.9)$ \\
$\quad$ Large bowel alone & 43 & $16(12.2)$ \\
$\quad$ Small and large & 115 & \\
$\quad$ bowel & & $30(11.9)$ \\
$\quad$ Total & 223 & $194(38.8)$ \\
All patients & 306 & \\
Controls & & $0(0)$ \\
$\quad$ No disease & 16 & $0(0)$ \\
Infectious colitis & 15 & $0(0)$ \\
Ischemic colitis & 1 & $0(0)$ \\
$\quad$ Total & 32 &
\end{tabular}

Location or extent of disease within the colon of patients with ulcerative colitis had no significant effect on the rate of detection of atypical p-ANCA (Table 1). Patients with colonic mucosal disease limited to the distal $60 \mathrm{~cm}$ or less had a $65.3 \%$ detection rate for atypical p-ANCA. In contrast, patients with more severe and extensive colitis, ie, over $60 \mathrm{~cm}$ of continuous mucosal disease extending proximally from the anal verge, had an atypical $\mathrm{p}$-ANCA detection rate of $69.8 \%$. In addition, the nature of the pathological process in ulcerative colitis, ie, presence or absence of endoscopically defined inflammatory pseudopolyposis, had no significant effect on the rate of ANCA detection.

Results from patients with Crohn's disease are also shown in Table 1. Most were negative for atypical p-ANCA. For the 30 Crohn's disease patients with a positive test result, 26 had colonic involvement; however, four had disease localized to the small bowel alone.In these patients,the histologi cal 


\begin{tabular}{|c|c|c|c|c|}
\hline Country & UC & CD & Control & Reference \\
\hline USA & 68 & 12 & 0 & 3 \\
\hline USA, Canada & 61 & 6 & 0 & 6 \\
\hline USA & 68 & & & 7 \\
\hline USA, Canada & 68 & & 3 & 12 \\
\hline USA & 76 & 8 & & 10 \\
\hline Norway & 27 & 0 & 0 & 15 \\
\hline Germany & 83 & 25 & & 8 \\
\hline Germany & 59 & 10 & & 9 \\
\hline France & 50 & & 0 & 16 \\
\hline Sweden & 50 & 8 & & 14 \\
\hline Netherlands & 79 & 13 & 9 & 11 \\
\hline Greece & 30 & & & 17 \\
\hline Denmark & 50 & 24 & & 20 \\
\hline Hong Kong & 32 & & 0 & 18 \\
\hline Canada & 66.3 & 11.8 & 0 & * \\
\hline
\end{tabular}

*Present study

detection of granulomas in biopsy or surgical specimens had no relationship to the presence or absence of atypical $\mathrm{p}$ ANCA.

Replication of ANCA studies: In 40 patients with IBD (ulcerative colitis or Crohn's disease), the presence of ANCA was evaluated for two or more time intervals separated by at least six months. For each patient, the same positive or negative result was obtained at each time interval; thus, in this study, no intra-observer error was evident. Because only one 'blinded' technologist performed the assay, interobserver error evaluation was not assessed.

\section{DISCUSSION}

This study evaluated detection rates of atypical p-ANCA in the sera from patients with IBD. Almost seven of 10 patients (ie, 66.4\%) with ulcerative colitis were positive. In contrast, only about one of 10 patients (ie, 11.9\%) with Crohn's disease were positive. Results in this prospective study, therefore, confirm observations reported in smaller series, particularly from other North American centres (Table 2). Our results, however, also extend these observations to a formal evaluation of fresh serum samples obtained from 500 patients with IBD, the largest number reported in any single study to date. The high disease specificity of this subclinical serological marker in most patients with idiopathic ulcerative colitis strongly suggests that ANCA may be intimately involved in the pathogenesis of this disorder (24). Detection of ANCA was also independent of several variables, including disease activity, severity, localization within the colon, or extent or treatment of colonic disease. This finding is consistent with observations in many $(3,8,11,20)$, but not all $(25)$, studies and suggests that the detection of atypical p-ANCA in idiopathic ulcerative colitis reflects the presence of a fundamental alteration in immune regulation rather than an epiphenomenon associated with active IBD, particularly within the colon.
A significant number of ulcerative colitis patients, about $30 \%$, did not have detectable ANCA in this series. Further studies are needed to determine whether this is indicative of different forms or causes of ulcerative colitis. Yang et al (26) have suggested that ulcerative colitis is a 'genetically heterogeneous' disease process, and the presence of 'ANCA-positive' and 'ANCA-negative' patients may reflect this heterogeneous inflammatory process in ulcerative colitis. Clearly, if this hypothesis were true, then evaluation and especially treatment of patients with ulcerative colitis may be made more complex. Clinical trials of new medications, particularly those that have been engineered to alter the inflammatory response, may be made more difficult to interpret if patients are not from a single 'reagent grade' category of ulcerative colitis (27).

Although the observations in earlier studies and the present report suggest the possibility of a blood test to support a diagnosis in IBD patients, the utility of this serological marker in patients with diarrheal diseases appears to be very limited. If positive, the test may be helpful in persuading the clinician to follow a patient carefully after an initial presentation with acute colitis, especially if an infectious or acute self-limited colitis is suspected. Our experience appears to confirm data reported elsewhere (6) that the ANCA test appears to be uniformly negative in patients with culture-positive enteric bacterial enteritis or colitis. In patients with proven IBD, the clinician must still depend on clinical and pathological features to differentiate ulcerative colitis from Crohn's disease, especially if surgical treatment for colonic disease is being contemplated (eg, such as pelvic pouch surgery). In the present study, many patients with ulcerative colitis were ANCA-negative; in addition, a significant number of patients with Crohn's disease, including Crohn's disease involving the colon alone, were ANCA-positive. If other reasons for detection of this serological marker have been excluded, such as concomitant primary sclerosing cholangitis, a concordant assay result might then be especially reassuring for an established diagnosis of ulcerative colitis or Crohn's disease. Conversely, a discordant assay result might lead to re-evaluation, but not necessarily exclusion, of the patient considering colectomy and creation of a pelvic pouch. Defining serological markers to differentiate patients with ulcerative colitis from Crohn's disease or to identify potential genetic risk factors, such as the microsatellite loci for different tumour necrosis factors (28), may eventually prove to be very useful for therapeutic studies in patients with IBD.

\section{REFERENCES}

1. Gross WL, Schmitt WH, Csernok E. ANCA and associated diseases: immunodiagnostic and pathogenetic aspects. Clin Exp Immunol 1993;91:1-12.

2. Hagen EC, Ballieux EPB, van Es LA, Daha MR, van der Woude FJ. Antineutrophil cytoplasmic autoantibodies: a review of the antigens involved, the assays, and the clinical and possible pathogenetic consequences. Blood 1993;81:1996-2002.

3. Saxon A, Shanahan F, Landers C, Ganz T, Targan S. A subset of antineutrophil anticytoplasmic antibodies is associated with inflammatory bowel disease. J Allergy Clin Immunol 1990;86:202-10.

4. Shanahan F, Landers C, Duerr R, Targan SR. Neutrophil autoantibodies as disease markers for ulcerative colitis. Immunol Res 1991;10:479-84.

5. Shanahan F. Neutrophil autoantibodies in inflammatory bowel disease: are they important? Gastroenterology 1994;107:586-9.

6. Duerr RH, Targan SR, Landers CJ, Sutherland LR, Shanahan F. 
Neutrophil autoantibodies in ulcerative colitis. Comparison with other colitides/diarrheal diseases. Gastroenterology 1991;100:1590-6.

7. Duerr RH, Targan SR, Landers CJ, et al. Neutrophil cytoplasmic autoantibodies: a link between primary sclerosing cholangitis and ulcerative colitis. Gastroenterology 1991;100:1385-91.

8. Siebold F, Weber P, Klein R, Berg PA, Wiedmann KH. Clinical significance of antibodies against neutrophils in patients with inflammatory bowel disease and primary sclerosing cholangitis. Gut 1992;33:657-62.

9. Rump JA, Scholmerich J, Gross V, et al. A new type of perinuclear antineutrophil cytoplasmic antibody (p-ANCA) in active ulcerative colitis but not in Crohn's disease. Immunobiology 1990;181:406-13.

10. Hardarson S, LaBrecque DR, Mitros FA, Neil GA, Goeken JA. Anti-neutrophil cytoplasmic antibody in inflammatory bowel and hepato-biliary diseases. High prevalence in ulcerative colitis, primary sclerosing cholangitis, and autoimmune hepatitis. Am J Clin Pathol 1993;99:277-81

11. Oudkerk-Pool M, Ellerbroek PM, Ridwan BU, et al. Serum anti-neutrophil cytoplasmic autoantibodies in inflammatory bowel disease are mainly associated with ulcerative colitis. A correlation study between perinuclear antineutrophil cytoplasmic autoanti-bodies and clinical parameters, medical, and surgical treatment. Gut 1993;34:46-50.

12. Shanahan F, Duerr RH, Rotter JI, et al. Neutrophil autoantibodies in ulcerative colitis: familial aggregation and genetic heterogeneity. Gastroenterology 1992;103:456-61.

13. Cambridge G, Rampton DS, Stevens TRJ, McCarthy DA, Kamm M, Leaker B. Anti-neutrophil antibodies in inflammatory bowel disease: prevalence and diagnostic role. Gut 1992;33:668-74.

14. Peen E, Almer S, Bodemar G, et al. Anti-lactoferrin antibodies and other types of ANCA in ulcerative colitis, primary sclerosing cholangitis and Crohns' disease. Gut 1993;34:56-62.

15. Zauli D, Baffoni L, Cassani F, et al. Antineutrophil cytoplasmic antibodies in primary sclerosing cholangitis, ulcerative colitis, and autoimmune diseases. Gastroenterology 1992;102:1088-95.

16. Reumaux D, Delecourt L, Colombel JF, Noel LH, Duthilleul P, Cartot A. Anti-neutrophil cytoplasmic autoantibodies in relatives of patients with ulcerative colitis. Gastroenterology 1992;103:1706.

17. Dalekos GN, Manoussakis MN, Goussia AC, Tsianos EV, Moutsopoulos
HM. Soluble interleukin-2 receptors, antineutrophil cytoplasmic antibodies, and other autoantibodies in patients with ulcerative colitis. Gut 1993;34:658-64.

18. Sung JY, Chan FKL, Hsu R, Liew CT, Lawton JW. Ulcerative colitis and antineutrophil cytoplasmic antibodies in Hong Kong Chinese. Am J Gastroenterol 1993;88:864-9.

19. Sung JY, Chan FKL, Lawton J, et al. Anti-neutrophil cytoplasmic antibodies and inflammatory bowel diseases in Chinese. Dig Dis Sci 1994;39:886-92.

20. Hertervig J, Wieslander J, Johansson C, Wiik A, Nilsson A. Anti-neutrophil cytoplasmic antibodies in chronic inflammatory bowel disease. Prevalence and diagnostic role. Scand J Gastroenterol 1995;30:693-8.

21. Bansi DS, Fleming KA, Chapman RW. Importance of antineutrophil cytoplasmic antibodies in primary sclerosing cholangitis and ulcerative colitis: prevalence, titre, and IgG subclass. Gut 1996;38:384-9.

22. Lennard-Jones JE. Classification of inflammatory bowel disease. Scand J Gastroenterol 1989;24(Suppl 170):2-6.

23. Duerr RH, Neigut DA. Molecularly defined HLA-DR2 alleles in ulcerative colitis and an antineutrophil cytoplasmic antibody-positive subgroup. Gastroenterology 1995;108:423-7.

24. Targan SR, Landers CJ, Cobb L, MacDermott RP, Vidrich A. Perinuclear anti-neutrophil cytoplasmic antibodies are spontaneously produced by mucosal B cells of ulcerative colitis patients. J Immunol 1995;155:3262-7.

25. Broekroelofs J, Mulder AHL, Nelis GF, Westerveld BD, Tervaert JWC, Kallenberg CGM. Anti-neutrophil cytoplasmic antibodies (ANCA) in sera from patients with inflammatory bowel disease (IBD). Relation to disease pattern and disease activity. Dig Dis Sci 1994;39:545-9.

26. Yang $\mathrm{H}$, Rotter JI, Toyoda $\mathrm{H}$, et al. Ulcerative colitis: a genetically heterogeneous disorder defined by genetic (HLA class II) and subclinical (antineutrophil cytoplasmic antibodies) markers. J Clin Invest 1993;92:1080-4.

27. Targan SR. New IBD markers: definition of disease heterogeneity. Can J Gastroenterol 1995;9:301-4.

28. Plevy SE, Targan SR, Yang H, Fernandez D, Rotter JI, Toyoda H. Tumor necrosis factor microsatellites define a Crohn's disease- associated haplotype on chromosome 6. Gastroenterology 1996;110:1053-60. 


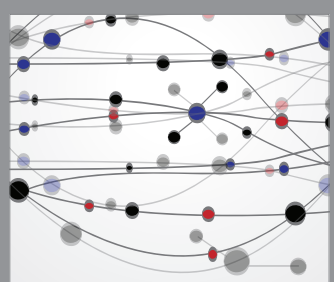

The Scientific World Journal
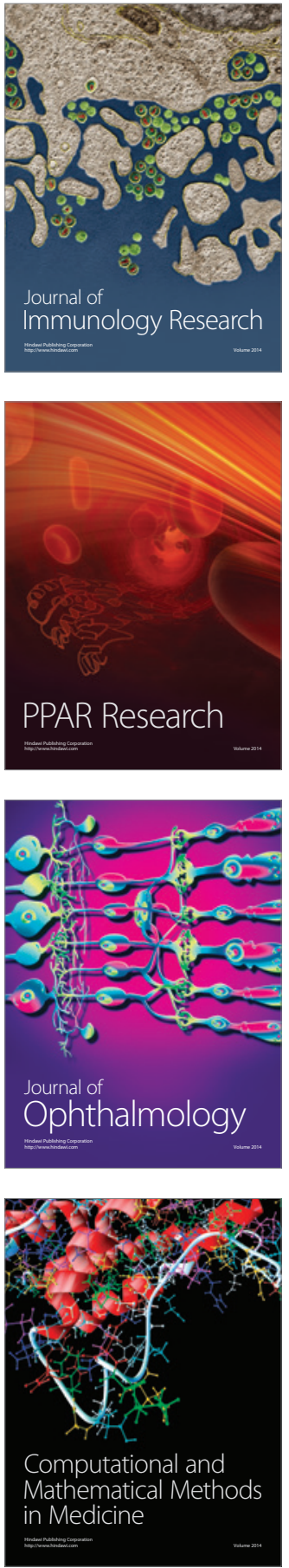

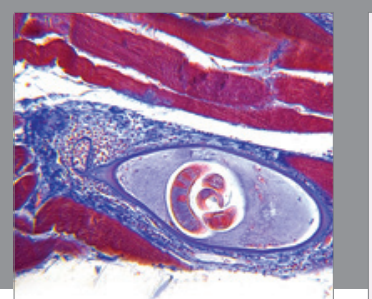

Gastroenterology Research and Practice

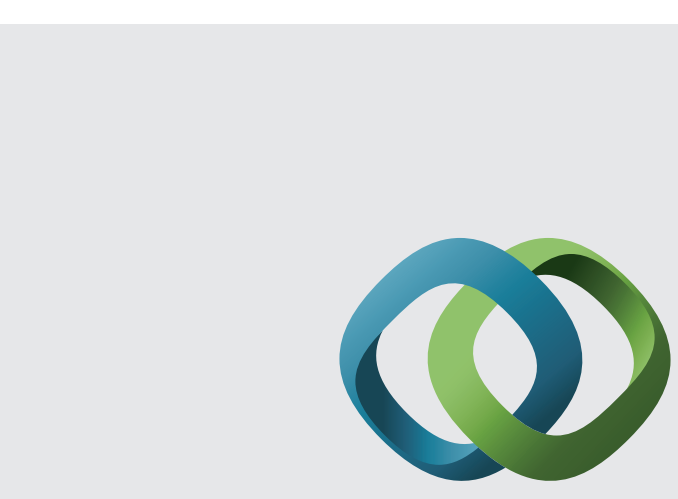

\section{Hindawi}

Submit your manuscripts at

http://www.hindawi.com
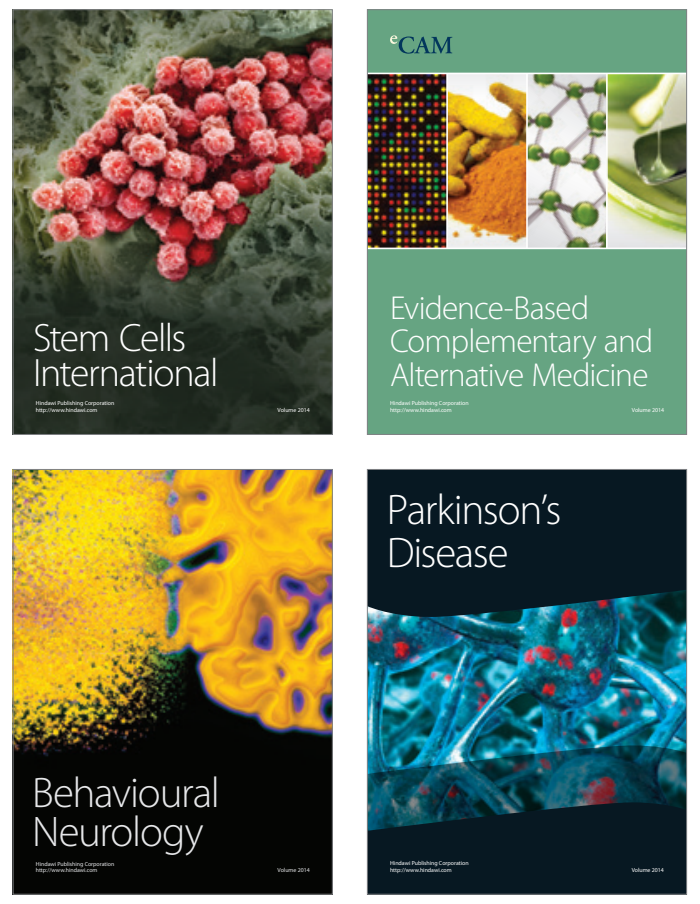
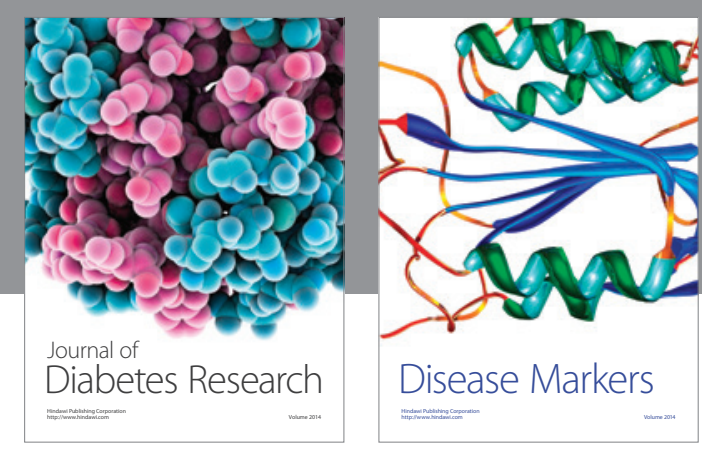

Disease Markers
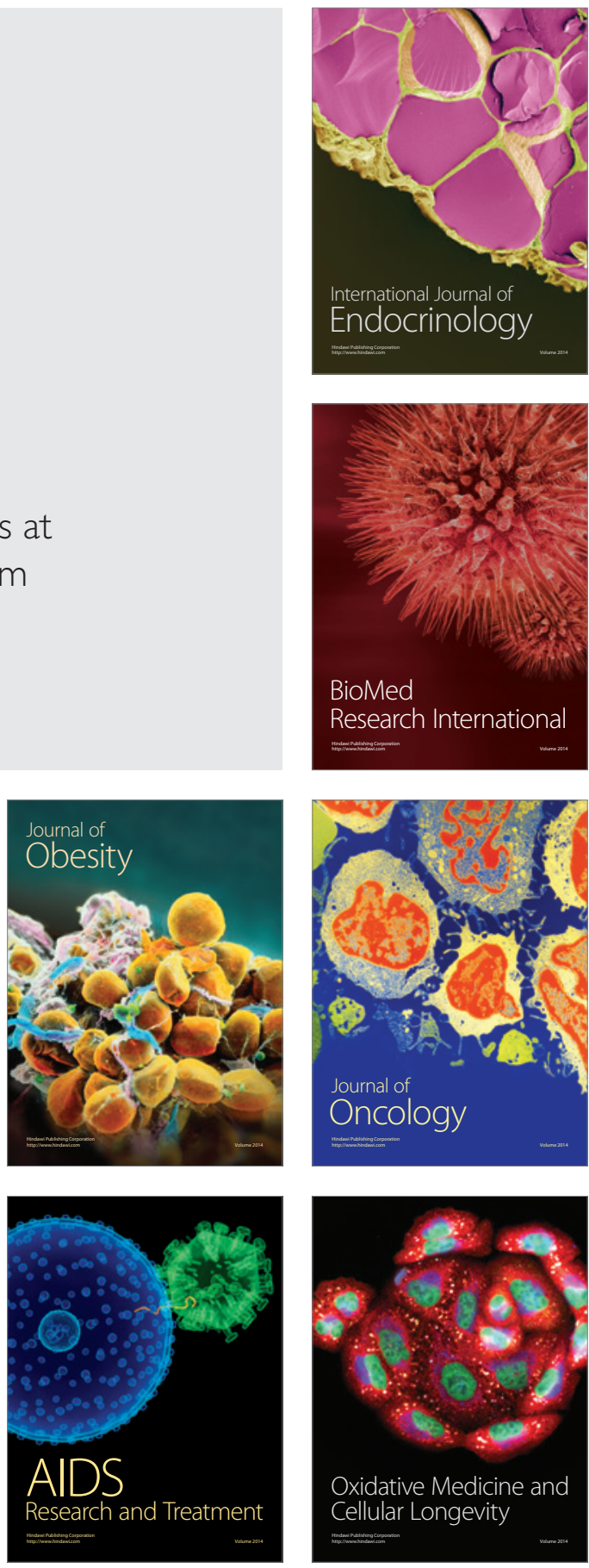